\title{
Abdominal Pregnancy
}

National Cancer Institute

\section{Source}

National Cancer Institute. Abdominal Pregnancy. NCI Thesaurus. Code C92921.

Ectopic pregnancy characterized by the implantation of the embryo in the peritoneal cavity or abdominal organs. 